\title{
Low-cost compact diffuse speckle contrast flowmeter using small laser diode and bare charge-coupled-device
}

\author{
Chong Huang \\ Myeongsu Seong \\ Joshua Paul Morgan \\ Siavash Mazdeyasna \\ Jae Gwan Kim \\ Jeffrey Todd Hastings \\ Guoqiang $\mathrm{Yu}$
}




\section{Low-cost compact diffuse speckle contrast flowmeter using small laser diode and bare charge-coupled-device}

\author{
Chong Huang, ${ }^{a}$ Myeongsu Seong, ${ }^{\text {a,b }}$ \\ Joshua Paul Morgan, ${ }^{c}$ Siavash Mazdeyasna, ${ }^{a}$ \\ Jae Gwan Kim, ${ }^{b}$ Jeffrey Todd Hastings, ${ }^{c}$ and \\ Guoqiang $\mathbf{Y u}^{\mathrm{a}, \star}$ \\ aUniversity of Kentucky, Department of Biomedical Engineering, \\ 143 Graham Avenue, Lexington, Kentucky 40506, United States \\ ${ }^{b}$ Gwangju Institute of Science and Technology, Department of \\ Biomedical Science and Engineering, 123 Cheomdan Gwagiro, \\ Bukgu, Gwangju 61005, Republic of Korea \\ 'University of Kentucky, Department of Electrical and Computer \\ Engineering, 512 Administration Drive, Lexington, Kentucky 40506, \\ United States
}

\begin{abstract}
We report a low-cost compact diffuse speckle contrast flowmeter (DSCF) consisting of a small laser diode and a bare charge-coupled-device (CCD) chip, which can be used for contact measurements of blood flow variations in relatively deep tissues (up to $\sim 8 \mathrm{~mm}$ ). Measurements of large flow variations by the contact DSCF probe are compared to a noncontact CCD-based diffuse speckle contrast spectroscopy and a standard contact diffuse correlation spectroscopy in tissue phantoms and a human forearm. Bland-Altman analysis shows no significant bias with good limits of agreement among these measurements: $96.5 \% \pm 2.2 \%(94.4 \%$ to $100.0 \%)$ in phantom experiments and $92.8 \%$ in the forearm test. The relatively lower limit of agreement observed in the in vivo measurements $(92.8 \%)$ is likely due to heterogeneous reactive responses of blood flow in different regions/volumes of the forearm tissues measured by different probes. The low-cost compact DSCF device holds great potential to be broadly used for continuous and longitudinal monitoring of blood flow alterations in ischemic/hypoxic tissues, which are usually associated with various vascular diseases. ๑ 2016 Society of Photo-Optical Instrumentation Engineers (SPIE) [DOI: 10.1117/1.JBO.21.8.080501]
\end{abstract}

Keywords: diffuse speckle contrast; charge-coupled-device; blood flow.

Paper 160281LR received May 2, 2016; accepted for publication Jul. 18, 2016; published online Aug. 8, 2016.

Blood flow (BF) is a critical factor affecting the delivery of oxygen and nutrition to and removal of wastes from tissue. The measurement of BF alteration in the tissue helps characterize many diseases represented with tissue ischemia and hypoxia such as cerebral vascular disease, peripheral artery disease, and cancer. Compared to large imaging modalities such as computed tomography, magnetic resonance imaging, and positron emission tomography for $\mathrm{BF}$ measurements, optical instruments are relatively portable, fast, continuous, and inexpensive. Optical techniques based on dynamic light scattering are the most common methods for $\mathrm{BF}$ measurements including laser speckle contrast imaging (LSCI) ${ }^{1}$ and diffuse correlation spectroscopy/tomography (DCS/DCT). ${ }^{2-5}$ Each technique, however, has key issues that limit its application. LSCI uses wide-field illumination and charge-coupled-device (CCD) detection of spatial laser speckle contrasts to achieve rapid highresolution two-dimensional mapping of $\mathrm{BF}$ in superficial tissues (depth $<1 \mathrm{~mm}$ ). By contrast, DCS/DCT uses coherent near-infrared (NIR) point-source illumination and single-photon-counting avalanche photodiodes (APDs) to accommodate spectroscopic or tomographic measurements of $\mathrm{BF}$ variations in deep tissues (up to $\sim 15 \mathrm{~mm}$ ). However, the use of expensive long-coherence length lasers and APDs limits its spatial-temporal resolution and increases instrumentation cost.

There have been a few recent technical advancements toward BF measurements in deep tissues with coherent NIR point-source illumination and high-sensitive CCD detection [e.g., electron multiplication CCD (EMCCD)], where the diffuse speckle contrast spectroscopy/tomography extracts deep tissue BF information using the relationship between diffuse speckle contrast parameters and DCS/DCT theory. ${ }^{6-8}$ Hundreds of detectors provided by the CCD significantly improve the spatial and temporal resolution and reduce the instrumentation cost and dimension. In these measurement setups, a CCD sensor is generally connected with optical lenses ${ }^{6-8}$ or optical fibers ${ }^{9}$ to detect diffuse speckle contrast variations induced by the motion of red blood cells in the tissue (i.e., BF). A long-coherence length laser ${ }^{6,8,9}$ or a small laser diode $^{7}$ are usually coupled to a lens system or an optical fiber to deliver NIR light to the target tissue. However, these noncontact (through optical lenses) or seminoncontact measurements (through optical lenses and optical fibers) with lasers and CCD cameras make the measurements very sensitive to motion artifacts and ambient light. In addition, EMCCDs and long-coherence length lasers used are still quite expensive and large. ${ }^{6,8}$

To overcome these limitations, we propose to develop and validate a low-cost compact diffuse speckle contrast flowmeter (DSCF) probe consisting of a small laser diode and a bare CCD sensor/chip (without lenses), which can be directly placed on the tissue surface for contact measurements of BF variations in deep tissues. Figure 1 shows the schematic of DSCF probe, which is placed on the surface of turbid media (e.g., tissue phantoms or human tissues) for flow measurements. A black foam pad was used to confine a small, inexpensive laser diode (L785P25, Thorlabs, New Jersey; wavelength: $785 \mathrm{~nm}$, power: $25 \mathrm{~mW}$, diameter: $5.6 \mathrm{~mm}$, price: $\$ 35$ ) driven by a current controller (LDC202C, Thorlabs, New Jersey, price: \$989) and a small bare CCD sensor chip (CMLN-13S2M-CS, Point Grey, BC, Canada; dimensions: $40 \times 32 \mathrm{~mm}^{2}, 1296 \times 964$ pixels, price: $\$ 300$ ), so that the source-detector (S-D) distance between the laser diode and CCD sensor can vary from 13 to $18 \mathrm{~mm}$ [Fig. 1(a)]. Based on photon diffusion theory, NIR light penetration depth in tissues is approximately one half of the S-D distance. ${ }^{2-5}$ The CCD chip is connected and controlled by a laptop through a universal serial bus cable.

One issue with the CCD-based contact measurement is the accumulated heat from the bare CCD sensor/chip, which is directly contacted to the surface of target media. To spread the 


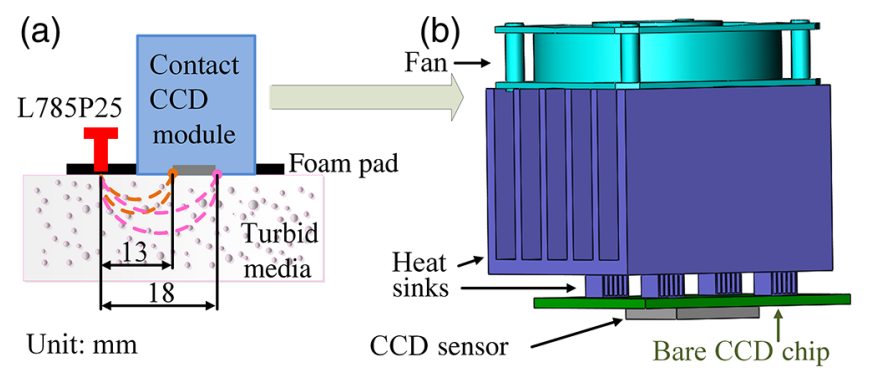

Fig. 1 (a) A compact DSCF probe placed on the surface of a turbid medium. (b) The DSCF probe consists of a small laser diode (L785P25) and a custom-made contact CCD module, which are confined by a black foam pad and covered by a transparent thin film (not shown) to prevent the CCD sensor/chip from being shortened by the liquid tissue phantom.

heat generated by the CCD chip, multiple small heat sinks (dimensions: $6 \times 6 \mathrm{~mm}^{2}$ ) were glued by thermal compound on the integrated circuits of the CCD chip. A $40 \times 40-\mathrm{mm}^{2}$ heat sink connected to a small silent fan was then glued on top of these small heat sinks for heat dissipation.

The laser diode L785P25 is inserted into a socket (S7060R, Thorlabs, New Jersey) and powered by a constant-current driver (LDC 205C, Thorlabs, New Jersey). The laser diode oscillates in a single transverse mode, but supports multiple longitudinal modes, and would not be expected to exhibit a long-coherence length. However, few mode lasers have complex correlation functions that are not well described by a single coherence length. To assess this, we placed L785P25 in a Michaelson interferometer and operated it under the same conditions as in the DSCF (constant 50-mA drive current with no active temperature stabilization). High-visibility ( $>0.8$ ) fringes were observed at many path-length differences between 0 and $400 \mathrm{~mm}$ (limit of test). Thus, the laser's coherence appears entirely sufficient for the S-D separations investigated here.

To calibrate/validate this DSCF probe (Fig. 1), we first conducted concurrent flow measurements on liquid tissue phantoms against a noncontact CCD-based diffuse speckle contrast probe ${ }^{6}$ and a standard contact DCS probe. ${ }^{4}$ The liquid tissue phantom is comprised of distilled water, India ink, and Intralipid, which has been commonly used for the calibration of flow measurement techniques. ${ }^{2,5,8}$ India ink is used to manipulate the absorption coefficient $\mu_{\mathrm{a}}$ while Intralipid provides particle Brownian motion (flow) and control of the reduced scattering coefficient $\mu_{\mathrm{s}}^{\prime}$. We set $\mu_{\mathrm{a}}=0.03 \mathrm{~cm}^{-1}$ and $\mu_{\mathrm{s}}^{\prime}=8 \mathrm{~cm}^{-1}$ at $785 \mathrm{~nm}$ to mimic the property of biological tissues. We created flow variations of Intralipid particles by changing the phantom temperature. The temperature of the phantom was initially set up to $70^{\circ} \mathrm{C}$ by an immersed heater (CH103, Ovente, California), and then decreased naturally until reaching the room temperature of $\sim 22^{\circ} \mathrm{C}$. A thermometer sensor (Physitemp, New Jersey) was placed inside the liquid tissue phantom for temperature measurements.

The first phantom experiment shown in Fig. 2(a) was designed to compare the results obtained concurrently from the contact measurements using two probes (i.e., DSCF and DCS) and a noncontact measurement using another CCD camera with optical lenses (FL3-FW-20S4M-C, Point Grey, BC, Canada). ${ }^{8}$ A long-coherence length DCS laser (DL785-100, CrystaLaser, Nevada; wavelength: $785 \mathrm{~nm}$, coherence length: $>5 \mathrm{~m}$, power: $100 \mathrm{~mW}$, dimensions: $135 \times 36 \times 30 \mathrm{~mm}^{3}$, price: $>\$ 7 \mathrm{~K}$ ) delivered NIR light to the liquid tissue phantom via a customized $1 \times 2$ fiber-based beam splitter (Fiberoptic Systems, California). A single-mode detector fiber (SM8005.6-125, Fibercore, California) connected to a four-channel APD module (SPCM-AQ4C, PerkinElmer, California; dimensions: $150 \times 130 \times 34 \mathrm{~mm}^{3}$, price: $\left.>\$ 11 \mathrm{~K}\right)$ and a four-channel autocorrelation board (price: $\sim \$ 5 \mathrm{~K}$ ) was used to collect the diffused light for the contact DCS measurement as a gold standard for comparisons. $.^{2-5} \mathrm{~S}-\mathrm{D}$ separation of $15 \mathrm{~mm}$ was used in all three probes [Fig. 2(a)]. Thirty data points (DCS)/frames (CCDs) were concurrently collected within 15 s (i.e., 2-Hz sampling rate) by the three probes at each of three different temperatures $\left(65^{\circ} \mathrm{C}, 45^{\circ} \mathrm{C}\right.$, and $\left.25^{\circ} \mathrm{C}\right)$. The two CCDs worked in parallel with the same exposure time of $5 \mathrm{~ms}$. Room light was turned off during these measurements.

For standard DCS data analysis, flow index was extracted by fitting the autocorrelation curve whose decay rate depended on the motion of moving Intralipid particles. ${ }^{4}$ For DSCF data analysis, the spatial speckle contrast $(K)$ over a selected window of $7 \times 7$ pixels was determined by calculating the ratio of standard deviation $(\sigma)$ and mean $(\mu)$ over these 49 pixels; i.e., $K=\sigma / \mu$. ${ }^{8}$ Flow-induced speckle fluctuation resulted in the reduction of laser speckle contrast in space for a given exposure time. Flow index was extracted via a nonlinear relationship between the $K$ and flow index under semi-infinite geometry. ${ }^{8}$ To increase the signal-to-noise ratio (SNR) of flow detection, a $3 \times 3$ adjacent pixel window array (covering an area of $\sim 78 \mu \mathrm{m} \times 78 \mu \mathrm{m}$ with nine values of $K$ were averaged representing one DSCF

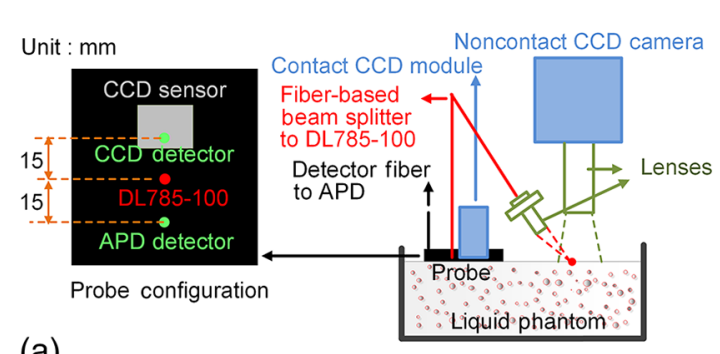

(a)

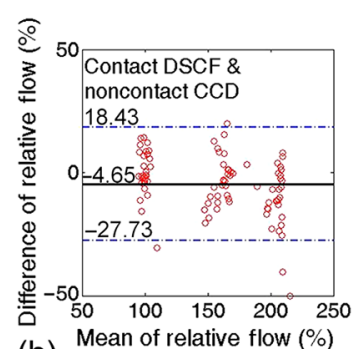

(b)

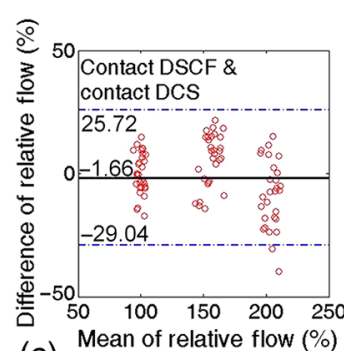

(c)

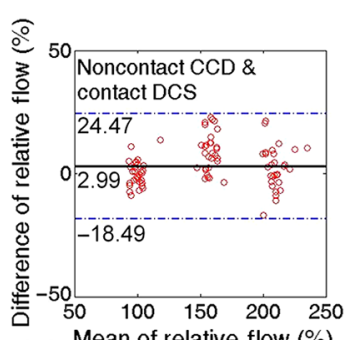

(d)

Fig. 2 Phantom experiments to calibrate/validate the contact DSCF probe against a noncontact CCDbased diffuse speckle contrast probe and a standard contact DCS probe. (a) Experimental setup (right) and the configuration (left) of contact DSCF and DCS probes. Note that all three probes (i.e., contact DSCF probe, contact DCS probe, and noncontact CCD-based probe) shared one long-coherence laser source (DL785-100). Bland-Altman plots with the limits of agreement (dashed lines) for the comparisons between relative flow changes measured by the (b) contact DSCF probe and noncontact CCD-based probe, (c) contact DSCF probe and standard contact DCS probe, and (d) noncontact CCD-based probe and standard contact DCS probe. 


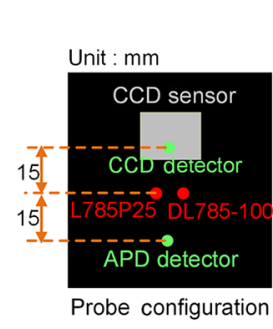

(a)

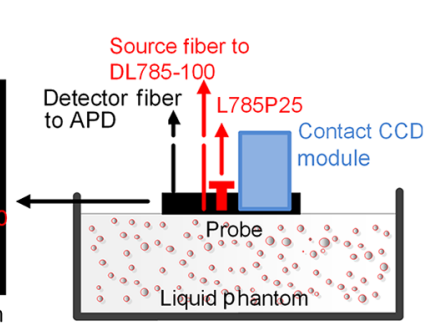

(b)

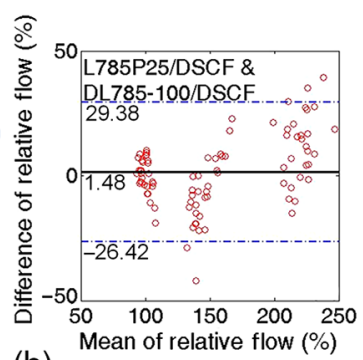

b)

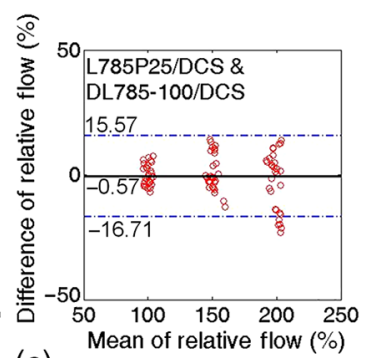

(c)

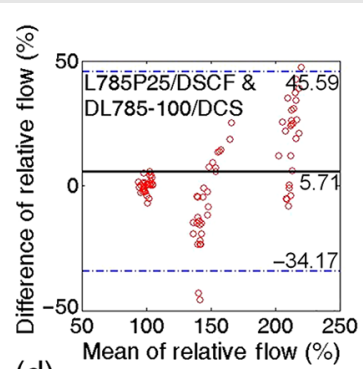

(d)

Fig. 3 Phantom experiments to compare the results obtained from two different laser sources: a small laser diode (L785P25) placed on the phantom surface versus a long-coherence DCS laser (DL785-100) coupled with an optical fiber. (a) Experimental setup (right) and configuration (left) of contact DSCF and DCS probes. Bland-Altman plots for the comparisons between relative flow changes measured by the (b) L785P25/DSCF and DL785-100/DSCF, (c) L785P25/DCS and DL785-100/DCS, and (d) L785P25/ DSCF and DL785-100/DCS.

detector. In this study, the detector center was chosen at $15 \mathrm{~mm}$ away from the laser source [Fig. 2(a)], allowing a penetration depth of $\sim 8 \mathrm{~mm}$ beneath the tissue surface. Relative flow changes were calculated by normalizing flow data at all measurement steps (i.e., $65^{\circ} \mathrm{C}, 45^{\circ} \mathrm{C}$, and $25^{\circ} \mathrm{C}$ ) to the averaged flow index (assigning $100 \%)$ at the last step $\left(25^{\circ} \mathrm{C}\right)$.

A Bland-Altman plot analysis was used to determine the agreement between different measurements and a MannWhitney u-test was used to test the systematic difference in mean values between the measurements (i.e., the fixed bias). Figures 2(b)-2(d) show Bland-Altman plots for the comparisons between the two of three flow measurements by the three optical probes. $Y$-axis shows the difference between the two paired measurements $(A-B)$ and $X$-axis shows the mean value of these measurements $(A+B) / 2$. The horizontal solid line represents the mean value of $(A-B)$ and horizontal dashed lines represent the mean values of $(A-B) \pm 1.96 \times$ standard deviation of $(A-B)$. For a good agreement between the two measurements, 95\% of the differences are expected to be less than 1.96 standard deviation. ${ }^{10}$ Good limits of agreement among these measurements [95.6\% in Fig. 2(b), 97.8\% in Fig. 2(c), and $100.0 \%$ in Fig. 2(d)] were observed. This experiment demonstrated that the contact and noncontact CCD-based diffuse speckle contrast measurements of flow changes generated highly consistent results [Fig. 2(b)], which also significantly agreed with the standard contact DCS measurement results [Figs. 2(c) and 2(d)].

The second phantom experiment shown in Fig. 3(a) was designed to compare the results obtained by the small laser diode (L785P25) for DSCF and the long-coherence laser (DL785-100) for standard DCS. Experimental protocols, data collection, and data analyses were similar to those described above. For each measurement at certain temperature, two lasers were turned on alternatively and 30 data points were collected in parallel by the DSCF and DCS detectors at the sampling rate of $2 \mathrm{~Hz}$.

Figures 3(b)-3(d) show the Bland-Altman analysis between the two of three flow measurements by different compositions of sources and detectors. Good limits of agreement among these measurements [94.4\% in Fig. 3(b), 94.4\% in Fig. 3(c), and $96.7 \%$ in Fig. 3(d)] were observed with no significant bias ( $p>0.05$ in Mann-Whitney u-tests). This experiment demonstrated that these two lasers (i.e., L785P25 and DL785-100) generated consistent results for the measurements of flow changes with both DSCF detector [Fig. 3(b)] and standard DCS detector [Fig. 3(c)]. The consistency between the DSCF (i.e., L785P25 and bare CCD module) and standard DCS (i.e., DL785-100 and
APD) measurements of flow changes [Fig. 3(d)] validated our new methodology (i.e., DSCF).

Further validation measurements in human tissues were approved by the University of Kentucky (UKY) Institutional Review Board. A healthy volunteer participating in the study was asked to sit and extend his right forearm on a table. A DSCF probe and a standard DCS probe were confined by a foam pad and taped on the surface of his right forearm for concurrent $\mathrm{BF}$ measurements [Fig. 4(a)]. An arterial cuff-occlusion (230 $\mathrm{mmHg}$ ) paradigm was applied on subject's right upper arm to induce significant BF changes in the right forearm. The occlusion protocol included 3-min baseline, 3-min cuff inflation, and 3-min recovery period following the cuff deflation. The sampling rates for both DSCF and DCS measurements were set as $1 \mathrm{~Hz}$ (lower than the $2 \mathrm{~Hz}$ in tissue phantom experiments) to improve SNRs of BF measurements in human tissues. Data analyses were similar to those described in the tissue phantom experiments. Relative blood flow (rBF) changes were normalized to the mean value of baseline flow data before the cuff-occlusion (assigning 100\%).

Figure 4(b) shows the time course rBF data measured concurrently by the DSCF and DCS probes in the forearm before, during, and after the occlusion. The relatively lower limit of

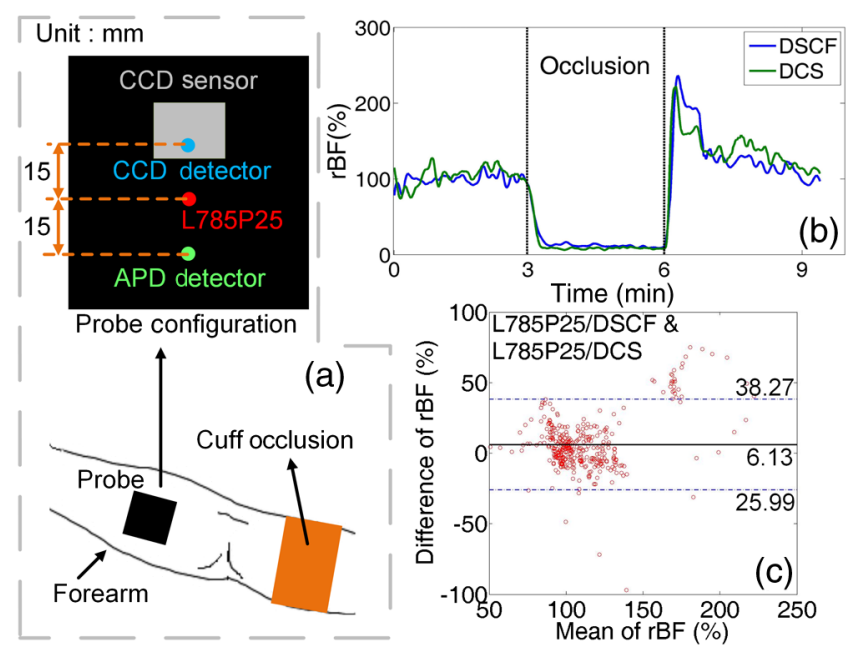

Fig. 4 In vivo experiments on a human forearm to compare the results obtained by DSCF and DCS probes. (a) Experimental setup (bottom) and the configuration (upper) of contact DSCF and DCS probes. (b) Forearm rBF responses during arterial occlusion measured by the DSCF and DCS probes. (c) Bland-Altman plots for the comparisons between $\mathrm{rBF}$ changes measured by the two probes. 
agreement observed in the forearm measurement [92.8\% in Fig. 4(c)], as compared to those in the phantom tests, was likely due to heterogeneous reactive responses of $\mathrm{BF}$ in different regions/volumes of the forearm tissue measured by different probes. No significant bias was observed between the two measurements ( $p>0.05$ in the Mann-Whitney u-test).

To summarize, we have designed a low-cost compact DSCF probe for BF measurements in relatively deep tissues $(\sim 8 \mathrm{~mm}$ depth). The cost-effective compact DSCF probe consists of a small laser diode and a bare CCD chip, which can be taped on top of the tissue surface allowing for contact measurements of BF variations in deep tissues. Sufficient speckle contrast is obtained by the DSCF probe for reliable flow measurements despite the more complex coherence properties of the laser diode. Measurements of flow changes by the contact DSCF probe are compared to a noncontact CCD-based diffuse speckle contrast probe ${ }^{8}$ and a standard contact DCS probe ${ }^{4}$ in tissue simulating phantoms and a human forearm. Bland-Altman analysis shows good limits of agreement among these measurements: $96.5 \% \pm 2.2 \%(94.4 \%$ to $100.0 \%)$ in the phantom experiments and $92.8 \%$ in the forearm test.

In contrast to noncontact CCD-based diffuse speckle contrast probes, ${ }^{6-8}$ the DSCF probe allows for contact measurements of tissue $\mathrm{BF}$ variations, which can avoid potential motion artifacts and ambient light influence occurred in noncontact measurements. The major difference between the noncontact $\mathrm{CCD}$ detection scheme and contact DSCF is the way to use CCD: the noncontact $\mathrm{CCD}$ detection scheme uses a CCD camera with lenses without touching the tissue whereas the contact DSCF uses a bare CCD chip in contact with the tissue surface. The noncontact CCD probe with a zoom lens provides the flexibility to change magnification and resolution of imaging, but should not affect the detection of rBF. Therefore, the principles of noncontact CCD detection scheme and contact DSCF are essentially the same. The consistent results from our noncontact and contact CCD measurements of flow variations [Fig. 2(b)] support our speculation. In addition, continuous measurements of $\mathrm{rBF}$ by the contact DSCF probe can be reproducible as long as the probe pressure on the measured tissue keeps constant. $^{3}$ Investigation of pressure influence on the measurements of absolute BF indices will be the subject of future study.

Compared to conventional contact DCS probes which commonly use rigid optical fibers for light delivery and detection, ${ }^{4}$ the compact DSCF probe can be placed directly on the tissue surface without any optical fiber. The connections between the DSCF probe and a control unit (including a driver for the laser diode and a laptop for CCD operation) are all electrical wires/cables, which provide flexibility for probe installation and offer potential for remotely longitudinal monitoring of tissue $\mathrm{BF}$ variations through wireless data transferring. Moreover, using the inexpensive laser diode and bare CCD chip, the cost of a DSCF is $\sim \$ 1,300$ and $\sim 18$ times lower than a conventional four-channel DCS flowmeter that uses a long-coherence laser, a four-channel APD module, and a four-channel autocorrelator board. The cost efficiency can be improved even more when conducting tomographic measurements as the single CCD chip of DSCF probe provides multiple detectors for image reconstruction. ${ }^{7,8}$ In addition, DSCF technique can be easily extended to measure, simultaneously, BF and oxygenation variations in deep tissues via adding another laser diode at different wavelengths (e.g., $\sim 854 \mathrm{~nm}$ ). ${ }^{4}$

A remaining challenge is how to effectively spread the heat generated by the CCD chip. In the present design, multiple heat sinks and a small fan are used to maintain the temperature of the CCD chip in a normal working range, which, however, makes the DSCF probe relatively large and inconvenient to install. We are currently exploring more efficient ways for heat dissipation (e.g., using a CMOS sensor with less heat load or a small semiconductor cooler for heat dissipation). We hope that with further technology improvement the low-cost compact DSCF probe can be further miniaturized and thus broadly used for continuous and longitudinal monitoring of $\mathrm{BF}$ alterations in ischemic/hypoxic tissues, which are usually associated with vascular diseases. For example, DSCF device may be used as a routine cerebral monitor for the management of critically ill neonates who are at highest risk for neurological morbidities. Ultimately, we expect to commercialize this noninvasive, fast, low-cost, and portable device for routine assessment and management of neonatal brain health.

\section{Acknowledgments}

The authors acknowledge funding support from the National Institutes of Health (NIH) R01-CA149274 (G. Yu) and R21AR062356 (G. Yu). The content is solely the responsibility of the authors and does not necessarily represent the official views of the NIH. We also acknowledge the support from UKY Reese S. Terry professorship (J. T. Hastings), Global PhD Fellowship Program NRF-2015H1A2A1032268 (M. Seong) and the Department of Biomedical Science and Engineering in the Gwangju Institute of Science and Technology (J. G. Kim), Republic of Korea. We also thank Dr. Ruigang Yang for lending us the CCD cameras.

\section{References}

1. D. A. Boas and A. K. Dunn, "Laser speckle contrast imaging in biomedical optics," J. Biomed. Opt. 15(1), 011109 (2010).

2. Y. Lin et al., "Three-dimensional flow contrast imaging of deep tissue using noncontact diffuse correlation tomography," Appl. Phys. Lett. 104(12), 121103 (2014).

3. T. Li et al., "Simultaneous measurement of deep tissue blood flow and oxygenation using noncontact diffuse correlation spectroscopy flowoximeter," Sci. Rep. 3, 1358 (2013).

4. Y. Shang et al., "Portable optical tissue flow oximeter based on diffuse correlation spectroscopy," Opt. Lett. 34(22), 3556-3558 (2009).

5. C. Huang et al., "Noncontact diffuse optical assessment of blood flow changes in head and neck free tissue transfer flaps," J. Biomed. Opt. 20(7), 075008 (2015).

6. R. Bi, J. Dong, and K. Lee, "Deep tissue flowmetry based on diffuse speckle contrast analysis," Opt. Lett. 38(9), 1401-1403 (2013).

7. H. M. Varma et al., "Speckle contrast optical tomography: a new method for deep tissue three-dimensional tomography of blood flow," Biomed. Opt. Express 5(4), 1275-1289 (2014).

8. C. Huang et al., "Speckle contrast diffuse correlation tomography of complex turbid medium flow," Med. Phys. 42(7), 4000-4006 (2015).

9. R. Bi, J. Dong, and K. Lee, "Multi-channel deep tissue flowmetry based on temporal diffuse speckle contrast analysis," Opt. Express 21(19), 22854-22861 (2013).

10. J. M. Bland and D. G. Altman, "Statistical methods for assessing agreement between two methods of clinical measurement," Lancet 1(8476), 307-310 (1986). 